\title{
Kreativität, Technizität und Autopoiesis. Zur Bedeutung des Homo faber für das Verständnis der frühesten Menschheitsgeschichte
}

\section{Shumon T. Hussain}

Die Paläolitharchäologie beschäftigt sich mit der Erforschung der Altsteinzeit, dem ältesten Abschnitt der Menschheitsgeschichte. Die Altsteinzeit ist vor allem durch das regelhafte Auftreten von Steinartefakten gekennzeichnet, die den Hauptanteil des verfügbaren Quellenmaterials ausmachen. Diese von den Vorfahren des heutigen Menschen und anderer Hominiden gefertigten technischen Objekte erlauben einen Einblick in die Evolution der 'herstellenden Tätigkeiten des Menschen. Es liegt daher auf der Hand, so möchte man meinen, dass das Konzept des Homo faber - pragmatisch gefasst der >arbeitende` oder `produzierende` Mensch - eine Schlüsselrolle für das Verständnis dieser menschheitsgeschichtlichen Epoche einnimmt. Während sich die Paläolitharchäologie dem faber-Topos insofern angenommen hat, dass sie immer wieder das `Erzeugerproblem von Artefakten in den Mittelpunkt der Fachdiskussionen rückt, ist der faber-Begriff auf theoretisch-konzeptueller Ebene bis heute weitgehend unterbestimmt geblieben. Das liegt nicht zuletzt wohl auch daran, dass die >Werkzeug-herstellende` Achse des Menschseins (man the tool-maker) der Forschung in der Regel als selbstverständlich und daher nicht weiter klärungsbedürftig erscheint.

Ausgehend von dieser Feststellung beleuchtet der vorliegende Beitrag die Bedeutung des Homo-faber Begriffs in der Paläolithforschung und versucht dessen erkenntnisleitendes Potenzial herauszuarbeiten. Geklärt werden soll dabei insbesondere, welche allgemeinen Interpretationsimpulse das faber-Konzept zu liefern vermag und welche Konsequenzen sich daraus für das Verständnis der frühen Menschheitsgeschichte ergeben. Zu diesem Zweck wird zunächst die Rolle des faber in der anglophon geprägten Diskussion um die behavioural modernity des Menschen in den Blick genommen. Diese wird dann dem faber-Begriff der französischen Technologie-Schule gegenübergestellt. Die exemplarische Untersuchung dieser beiden Diskursfelder zeigt, dass ein differenzielles faber-Verständnis die Perspektive auf die Evolution des altsteinzeitlichen Menschen nachhaltig verändern kann. 


\section{Der negativ bestimmte Homo faber der behavioural modernity Debatte}

Sowohl Paläoanthropologie als auch Paläolitharchäologie haben sich in den letzten Jahrzehnten insbesondere mit der Frage nach der »Modernität« des Menschen beschäftigt (vgl. Mellars/Stringer 1989; Stringer 2006; Knight 2010). Das erklärte Ziel dieser Auseinandersetzung, die vor allem die englischsprachige Forschung weitgehend bestimmt hat, ist

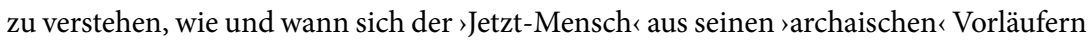
evolutionär herausgebildet hat (Watts 2010). Der Dreh- und Angelpunkt dieser Debatte ist daher naturgemäß der `moderne`Mensch, so wie er im `Hier und Jetzt ‘ gegeben ist. Dieser Jetzt-Mensch wird in der Literatur gemeinhin mit dem Homo sapiens gleichgesetzt. ${ }^{1}$ Das Kernproblem der Debatte ist demgemäß die archäologisch überzeugende Unterscheidung zwischen modernen Menschen und deren Vorläuferformen wie z.B. den Neandertalern (vgl. Nowell 2010). Die Dichotomisierung zwischen `modern und `archaisch $`$ hat sich dabei zu einem Forschungsparadigma entwickelt.

Weil der moderne Mensch typischerweise als sapiens bestimmt wird, ist die gesuchte differentia specifica traditionell im Bereich des Kognitiven vermutet worden (vgl. Mithen 1998; Renfrew 2009). ${ }^{2}$ Die resultierende Forschungsfrage kann wie folgt formuliert werden: ,Wie kann die theoretisch-reflexive, logozentrische und erkenntnisorientierte Kognitionsbegabung des modernen Menschen anhand der materiellen Hinterlassenschaften der Altsteinzeit aufgespürt werden? $`$ Jene `Modernität` des Menschen müsste jedenfalls in einem >distanzierten Verhalten zur Umwelt erkennbar werden. Robert Kelly (2016: 36) formuliert das wie folgt:

»Es ist unsere Kapazität für Kultur, die Fähigkeit die Welt anders zu sehen, die Menschen von den anderen Primaten unterscheidet. Anthropologen diskutieren, wann Menschen diese Fähigkeit erlangten, wenn man aber die vorbeiziehende Welt der Hominiden von einem Hochsitz im Weltraum aus beobachtet, kann man nicht anders als festzustellen, dass etwas Entscheidendes sich zwischen 200.000 und 50.000 Jahren vor heute verändert hat. Irgendwann wurden Hominiden zu kulturellen Wesen, zu Menschen wie wir sie kennen. Das ist der Zeitpunkt, an dem wir die Fähigkeit zum religiösen Denken entwickelten, an dem wir Geschichten erzählen sowie Metaphern und Analogien zu nutzen lernten, an dem wir Wissenschaft, Kunst, Musik und Poesie kreieren konnten, und an dem wir die Fähigkeit entwickelten emotional auf Reden und Lieder zu reagieren« (Übersetzung d. Verf.; Hervorhebung im Original).

1 Zur Definition des Homo sapiens, siehe z.B. Grawe (1973a).

2 Dieses Spannungsverhältnis wird durch die Terminologie der Paläoanthropologie in gewisser Hinsicht schon vorweggenommen. Während der anatomisch moderne Mensch als Homo sapiens bezeichnet wird, sind seine Vorfahren unter Bezeichnungen wie Homo ergaster (der >arbeitende` Mensch) und Homo habilis (der >geschickte` Mensch) bekannt. Die evolutionäre Dualität von `Körper` und `Geist` schwingt bei dieser Begriffswahl immer schon mit. 
Diese sapiens-zentrische Perspektive führt unweigerlich zu einer erkenntnisleitenden Auftrennung von Kognition und Technik. Der Bereich der Technik scheidet schon allein deshalb als erklärende Komponente der menschlichen `Modernität ‘ aus, weil die Bearbeitung und Nutzung von Steingeräten einer ganzen Reihe von Hominiden zugerechnet werden kann - sie eignet sich deshalb nicht als Unterscheidungsmerkmal. Sogar heute lebende Menschenaffen verfügen offenkundig über die Fähigkeit einfache Steinwerkzeuge herzustellen (vgl. insb. Toth et al. 1993). Der >Modernität ‘ des Menschen wird deshalb meist über eine eigenständige Kategorie materieller Hinterlassenschaften nachgegangen. Diese Kategorie ist vielfach mit Bezeichnungen wie »symbolische« und »spirituelle Kultur « versehen worden (vgl. Renfrew/Morley 2009). Angenommen wird dabei stets, dass sich die kognitive Modernität des sapiens, welche über die rein technisch-instrumentell vermittelte Lebensweise anderer Hominiden hinausgeht, in der Herstellung und Nutzung neuartiger `über-technischer Objekte archäologisch niederschlägt.

Die prominentesten Beispiele für solche `besonderen` Objekte sind persönliche Schmuckstücke wie Perlen, durchlochte Tierzähne und Anhänger sowie allerlei gravierte und dekorierte Artefakte, die gemeinhin als mobile oder stationäre Kunstobjekte beschrieben werden (vgl. Conard 2010). Diese Objekte, so die gängige Deutung, zeigen einen neuartigen Weltbezug an, der ganz wesentlich >symbolisch-vermittelt ist (vgl. Knight/Power/Watts 1995; Mellars/Boyle/Bar-Yosef 2007) - Paul Mellars (1991) spricht in diesem Zusammenhang sogar von einer Human revolution. Der entsprechende Zugang zum Quellenmaterial weist Homo sapiens und Homo symbolicus jedenfalls als weitgehend deckungsgleich aus (Henshilwood/d'Errico 2011). Der moderne Mensch, so kann im Anschluss an Eliade (1959: 87, 95) und Cassirer (2007: 51) gesagt werden, schafft "symbolische Formen « und lebt in »symbolisch-religiösen Ordnungen «. Die >Modernität « des Menschen wird folgerichtig als Überwindung des >Tierzustands $\triangleleft$ bestimmt.

Die Konsequenz dieser Rekonstruktionslogik, die vor allem auf evolutionäre Brüche scharfstellt, ist die negative Charakterisierung des `herstellenden Menschen`. Der Homo faber wird vorrangig in seiner >Brückenfunktion ‘ bestimmt, sodass die spezifische Technikaffinität des Menschen als missing link zwischen werkzeugnutzenden Tieren und in symbolischen Welten agierenden Sapienten plausibel gemacht werden kann. Die Diskussion um den Status des sog. Oldowan, einer frühen Steinartefaktindustrie aus Afrika, kann hier als einschlägiges Beispiel angeführt werden (vgl. Toth/Schick 2009). Der steinartefakteherstellende Mensch der Altsteinzeit wird in der Englisch-sprachigen Fachdebatte demgemäß häufig aus dem Blickwinkel der $>$ Natur $<$ betrachtet. Lithische Technologie gilt als materielle Erweiterung des biophysischen Körpers und erlaubt so - qua »extrasomatische« Adaptation - die Erschließung neuer ökologischer Nischen (Kelly 2016: 28f., 32). Nicholas Toth und Kathy Schick (2009: 299) sprechen explizit von "synthetischen Organen «. Der Begriff des faber - falls er überhaupt explizit verwendet wird - thematisiert so vor allem den Übergang von der `Natur $<$ zur $\gg$ Kultur $<$ des Menschen. Der Mensch als faber erscheint evolutionär damit immer als `unvollständig‘

Diesem negativ bestimmten Homo faber der `Modernitäts-Debatte`, welcher vor allem als entwicklungsgeschichtliche >Vorstufe « des Homo symbolicus in den Blick gerät, kann ein substantieller und `Eigenpotenzialität $<$ betonender faber-Begriff gegenübergestellt werden. Wie im Folgenden genauer beleuchtet werden soll, erweist sich dieser vor 
allem für die Lithikforschung französischer Prägung als wegweisend und hat dort ein eigenständiges Diskursfeld begründet.

\section{Bergsons positiv bestimmter Homo faber}

Obwohl sich offenkundig eine lange Liste von Autoren um die substanzielle Bestimmung des Homo faber bemüht hat (vgl. z.B. Grawe 1973b), sind für den vorliegenden Beitrag vor allem die Arbeiten Henri Bergsons von Bedeutung. Nicht nur ist Bergsons zum Teil erheblicher Einfluss auf die französischsprachige Technik- und Paläolithforschung heute gut belegt (Audouze 2002; Schlanger 2004, 2015; Delitz 2015: 246-251), er hat sich zudem um eine evolutionstheoretische Erweiterung des faber-Begriffs verdient gemacht und das Konzept damit entscheidend dynamisiert. Bergsons faber-Konzeption ist untrennbar mit seiner Evolutions- und Zeittheorie verbunden, welche letztlich den Versuch unternimmt, die variablen Formationen des `Seins « über zugeordnete Prozesse des `Werdens $`$ verständlich zu machen. Sein faber-Begriff dient nicht zuletzt diesem Unterfangen.

Der Homo faber wird von Bergson in L'Évolution créatrice (1944 [1907]) eingeführt. Der Titel dieser Arbeit ist programmatisch. Bergson geht es vor allem darum, das `mechanistische Weltbild, so wie es von Descartes, Newton und zuletzt Darwin geprägt worden ist, zu überwinden, um Raum für das `Nicht-Notwendige`, >Kreative` und `Organische in der Evolution zu schaffen. Damit einher geht eine raffinierte Neubestimmung des Konzepts der `Zeit` sowie eine eingehende Beschäftigung mit dem Phänomen der `Dauer (durée). Insgesamt kann Bergsons Ansatz als ein Versuch gelesen werden, das zeitliche Wesen von Entwicklungsprozessen - verstanden als ein fortwährendes `Sich-Verändern und $`$ Entfalten $\prec$ - theoretisch ernst zu nehmen.

Bergsons Homo faber ist vor allem ein Gegenbegriff zum Homo sapiens, der als ein Produkt der Hybris des Jetzt-Menschen kritisiert wird (Bergson 1944 [1907]: 153). Nach Bergson verstellt eine Bestimmung des Menschen als sapiens den Blick auf das Wesentliche und `Sich-konstant-verändernde` in der Humanevolution: die Technizität. Die Idee des sapiens überhöht für Bergson unweigerlich die `theoretisch-szientistische` Dimension des Menschseins. Er entlarvt die Charakterisierung des Menschen als 'vernunftbegabtes Wesen mit der Befähigung zum >wissenschaftlichen Erkennen` damit als unzulässige Rückprojektion und problematisiert diese im Zusammenhang des evolutionären >Werdens der Menschheit. Zudem impliziert der Begriff des sapiens einen radikalen Bruch zwischen >Animalität $\triangleleft$ und `Humanität , weil nur letztere über einen erkennenden Logos verfügt. Das sapiens-Verständnis mit seinem Fokus auf das `Sein « ist deshalb zu statisch, um der evolutionären `Entfaltung \ des Menschen gerecht zu werden. Es scheitert nicht zuletzt am Versuch, die Besonderheit des Menschen schlüssig aus der Kontinuität von Mensch und Tier abzuleiten. Bergson konstatiert deshalb, dass die mit Hilfe des sapiens thematisierte menschliche Kognitivität wohl eher ein abgeleitetes als ein ursprüngliches Humankriterium ist. Die im Homo faber angelegte >Technikbegabtheit des Menschen schafft demgegenüber ein evolutionäres Entwicklungspotenzial, das der Kognitivität des sapiens logisch vorgelagert ist und zudem `genetisch $>$ zu erklären vermag. Das Potenzial für neuartige kognitive Weltzugänge muss nach Bergson deshalb vor allem in der `Dauerhaftigkeit des faber angelegt sein. 
Der Bergsonsche Homo faber folgt so dem Bestreben, evolutionäre Kontinuität und Differenz gemeinsam zu denken. Nach Bergson sind sowohl Mensch als auch Tier intelligente Werkzeugnutzer. Der Werkzeuggebrauch der Tiere ist jedoch auf deren Körperlogik eingestellt - Tiere nutzen entweder `körpereigene` Instrumente oder erweitern die Möglichkeiten ihrer Körpernutzung mit Hilfe von körperkomplementären Hilfsmitteln. ${ }^{3}$ Menschen hingegen können auf ‘körperunabhängige` Werkzeuge (Werkzeuge sensu stricto) zurückgreifen. Der Werkzeuggebrauch der Tiere bleibt deshalb weitgehend körpergebunden und den Notwendigkeiten des tierischen Lebens verhaftet, während Menschen über eine gewisse schöpferische Freiheit verfügen ihre Werkzeuge zu gestalten und zu (re)kombinieren. Die körperunabhängigen Werkzeuge des Menschen begründen eine materielle Eigenlogik, die sich modulierend auf Kognitionsvorgänge auswirkt. Die Werkzeuge des Menschen wirken deshalb auf die Verfassung von Körper und Geist zurück. Der menschliche Werkzeuggebrauch, im Unterschied zum Instrument-Gebrauch, lässt so Raum für Veränderung, Kreativität und Innovation - für die Entstehung von Neuem und Nichtvorhersagbarem.

Es ist dieses poietische Moment menschlichen Produzierens, das Bergson mit dem Homo faber einzufangen versucht. Menschliche Kognition ist demnach zuallererst in der `Produktionsintelligenz` (intelligence-de-produire) des faber begründet, aus der sich die theoretisch-reflexive Intelligenz des sapiens erst herausgebildet hat. Das >Wissen, wie $<$ (savoir-faire) ist dem `Wissen, dass (savoir-être) sowohl logisch als auch entwicklungsgeschichtlich vorgelagert. Der Homo sapiens gerät damit als eine Art evolutionäre Verdichtung des Homo faber in den Blick - im sapiens zeigt sich unsere faber-Natur, und nicht umgekehrt. Die poietische Technizität des Menschen wird als Quelle seiner bemerkenswerten Kognitionsfähigkeit erkennbar. Bergsons faber-Konzeption versucht dabei nicht zuletzt die Dualität von Geist und Materie zu überwinden. Geist ist für Bergson immer schon in Materie angelegt und bestimmte Materie-Konfigurationen realisieren schlichtweg bestimmte Formen von Geist (vgl. Bergson 1944 [1907]: 225f).

Bergsons Homo-faber-Begriff erlaubt somit die Untersuchung der phylogenetischen Kopplung von Denken und Herstellen. Die durch diese Kopplung freigesetzten Entwicklungsdynamiken autorisieren eine Rekonstruktion der kognitiven Vermögen des Menschen, ausgehend vom Faktum der Technizität. Anstatt den faber als etwas zu Überwindendes zu konzipieren, betont diese Perspektive die Möglichkeiten und Potenziale der technischvermittelten `Lebensform〈Mensch. Werkzeugherstellende Tätigkeiten kultivieren einen kognitiven Überschuss, der Neues herausfordert und Entwicklung fördert. Es ist dieses evolutionäre Primat des faber, das, wie der Verfasser im Folgenden zu zeigen versucht, die französische Paläotechnologie-Forschung bis heute antreibt.

3 François Sigauts (2012: 92-98) Unterscheidung zwischen outils-prothèses und outils vrais kann als eine Ausarbeitung dieser Konzeption gelten. 


\section{Der erweiterte Homo-faber-Begriff als Ausgangs- punkt der französischen Technologieschule}

\section{Faber-Ouvertüre: Leroi-Gourhan und Tixier}

Der Grundimpuls der französischen Paläolithforschung, die Menschwerdung vorwiegend aus der Perspektive des Homo faber verständlich zu machen, geht vor allem auf den Einfluss von André Leroi-Gourhan zurück. Leroi-Gourhan ist maßgeblich dafür verantwortlich zu machen, dass sich die Philosophie Henri Bergsons, und damit auch sein Konzept des faber, im intellektuellen Substrat der französischen Forschung festgesetzt hat (vgl. insb. Leroi-Gourhan 1949, 1952, 1953). Die vielen "Bergsonismen« des Parisers beschäftigen die Forschung bereits seit langem (vgl. Schlanger 2004, 2015). Auch wenn hier nicht im Detail auf das Wirken von Leroi-Gourhan eingegangen werden kann, sollte aber zumindest darauf hingewiesen werden, dass sein Verhältnis zu Bergson und $\mathrm{zu}$ dessen faber-Konzept als ambivalent eingestuft werden muss. Ausgehend von einer Fehlinterpretation von Bergsons faber (vgl. Leroi-Gourhan 1952), gelangt Leroi-Gourhan am Ende zu einer Verfeinerung des Konzepts ganz im Sinne Bergsons. ${ }^{4}$ Es ist vor allem dieser >richtig ` verstandene faber, der die französische Paläotechnologie-Forschung seitdem fruchtbar unterfüttert.

Bereits in Évolution et techniques (Leroi-Gourhan 1943/1945) wird die >Irreduzibilität‘ und >Vorrangigkeit`der Technizität in der Humanevolution betont. Für Leroi-Gourhan (1953, 1984 [1964/1965]) ist dabei das evolutionäre Wechselspiel zwischen Hand und Cerebrum von entscheidender Bedeutung. Das Schlüsselkonzept ist die `Externalisierung des Werkzeuggebrauchs, die sich erst aus der Evolution des menschlichen Körperbaus rekonstruieren lässt (vgl. Leroi-Gourhan 1984 [1964/1965]: 82, 319f.). Diese Externalisierungstheorie macht vor allem auf sich verändernde Bedingungen evolutionärer Entwicklungspfade aufmerksam. Demgemäß mündet die physisch-körperliche Evolution des Menschen im aufrechten Gang und damit in der Freistellung der opponierten Hand. Die Ablösung des Handapparats von den Aufgaben der Lokomotion stellt dabei die Weichen für eine sprichwörtliche >Befreiung « der menschlichen Lebensform (vgl. Johnson 2011). Sie leistet der Objekt-Manipulation Vorschub und eröffnet somit neue, weitgehend sunbedingte`Möglichkeiten der Weltbearbeitung. Die `Befreiung` von Hand und Geste beim Menschen fällt deshalb mit dem Übergang von der natürlichen Evolution, die weitgehend >deterministisch-mechanistischen Prinzipien folgt, zur kulturellen Evolution, die immer auch `kreativ-poietischen Charakter hat, zusammen.

Die manipulative, serzeugnisschwangere Hand bringt zudem eine eigenständige, Resonanz erzeugende >Artefakt-Welt ` hervor und verändert so die Dynamik der kognitiven Bezugnahme auf die Umwelt (vgl. insb. Lemonnier 2012). Das Konzept der 'Operationskette` (chaîne opératoire), welche Leroi-Gourhan dem ideellen Korpus seines Lehrers Mauss entlehnt, stützt diese Sichtweise. Operationsketten sind immer schon

4 Leroi-Gourhan gelangt trotz scharfer Kritik an Bergson zu einem ganz ähnlichen Ergebnis: er unterstreicht die `Ko-Implikation`von Technik und Kognition in der Humanevolution (vgl. Audouze 2002: 288). 
Vermengungen von kognitiven, sozialen und somatischen Fakultäten und beschreiben als solche `psycho-motorische s sowie `kulturstiftende Prozesse (vgl. Mauss 2012 [1935]: 390-392; Mauss in diesem Heft). Operationsketten können so als »rekursive Kulturtechniken « verstanden werden (Schüttpelz 2006), die sich aus dem `Freiheitspotenzial des faber speisen und externe Abhängigkeiten transzendieren. Wie Hand und Operationskette jeweils artikuliert sind, aber auch welche Gestalt eine Operationskette im konkreten Fall annimmt, ist natürlich ganz erheblicher Variabilität unterworfen. Dennoch gilt, dass die technisch-praxeologische Dimension von Operationsketten den in ihnen simprägnierten sozio-kognitiven Größen vorgängig ist (ebd.: 7-10; Heilmann 2016).

Weil Hand und Operationskette zudem über technische Gesten synchronisiert werden, erweist sich menschliche `Technizität ‘ als eine Frage des `Ineinandergreifens ` von Körpertechniken und Produktionstechniken (vgl. Balfet 1975, 1991; Bril 1984; de Beaune 2000). ${ }^{5}$ Dieses Verständnis des >Produzierens betont die bereits thematisierte praktische Intelligenz (savoir-faire) des Menschen und stützt zudem eine inklusive Lesart des faber, mit Hilfe derer alle steinartefakteproduzierenden Hominiden und `Frühmenschen`gleichermaßen mit berücksichtigt werden können - Jacques Pelegrin (1991) folgend kann an einer `Geschichte der praktischen Intelligenz` in der longue durée gearbeitet werden.

Dieser Grundgedanke ist bereits in Le geste et la parole angelegt, wo Leroi-Gourhan (1984 [1964/1965]) den Versuch unternimmt die einzigartige Sprachfähigkeit des Menschen aus der Technizität herzuleiten. Gestische Freiheit sowie die Entwicklung einer praktischen Intelligenz im Umgang mit `Artefakt-Welten ‘ werden dabei als Möglichkeitsbedingungen der Sprachevolution identifiziert. Diese Interpretation betont die evolutionäre Kontinuität von Sprache und Technik und legt das Hauptaugenmerk auf die Interaktion von Mensch und Materie, um die Genese des Jetzt-Menschen nachzuvollziehen. Sprache und symbolische Kognition werden als notwenige Konsequenz der faber-Natur des Menschen, nicht etwa als dessen Überwindung gedeutet. Erst die Kultivierung praktischer Intelligenz schafft die kognitiven Entwicklungspotenziale, die unserer `Sprachfähigkeit und `Symbolbegabtheit` zu Grunde liegen.

Die institutionelle Herausbildung der Technologie préhistoire, dem Nährboden der heutigen `Technologie-Schule`, in den frühen 1980er Jahren in Paris (vgl. Tixier 1980; Pigeot 1991) entspricht einem Mandat zur Erforschung dieses positiv gefassten Homo faber. Die Untersuchung der Menschheitsgeschichte meint seitdem vor allem die Erforschung ihrer technikgeschichtlichen Aspekte. Der französische Begriff technologie verweist seitdem keinesfalls mehr nur auf einen Untersuchungsgegenstand, wie er etwa im englischen Terminus technology angelegt ist, sondern bezeichnet eine eigenständige Disziplin, die sich dem Studium der menschlichen Technizität in all ihren Facetten und historischen Formatierungen verschrieben hat (vgl. Haudricourt 1987) - eine Forschungstradition, die sich mindestens bis zu Leroi-Gourhans Ansatz einer interdisziplinären technologie comparée zurückverfolgen lässt (vgl. de Beaune 2011).

Die epistemologische Schlüsselrolle des Homo faber für diese noch junge >TechnologieSchule « spiegelt sich nicht zuletzt auch in den Schriften eines ihrer Mitbegründer, Jacques Tixier, wider. In Méthode pour létude des outillages lithiques hält dieser gleich zu Beginn fest:

5 Zum Begriff der »technischen Geste«, siehe Bril/Roux (2002). 
»Der Begriff `faber « ist für mich untrennbar mit dem Begriff `Homo « verbunden. Ich gehöre zu denjenigen, die davon ausgehen, dass wir uns ab dem Moment, indem intentional, und deshalb geplant geschlagene harte Steingeräte auftreten bereits an der Schwelle der Hominisation befinden. Jenseits dessen liegt das, was Prähistoriker gemeinhin das Problem des `Menschen` nennen« (Tixier 2012 [1978]: 39; Übersetzung d. Verf.).

Die mit Tixier verknüpfte stechnologische Revolution ` der 1980er und 1990er Jahre geht dabei keinesfalls zufällig mit einer Schwerpunktverschiebung von den >Was-Fragen $<$ auf die ,Wie-Fragen ` der Technikforschung einher (Tixier 2012 [1978]: 33). Das übergeordnete Ziel der Untersuchung von Steinartefakten ist nicht länger deren finale Morphologie und Funktionalität zu erhellen, sondern die Organisation von lithischen Produktionsvorgängen zu rekonstruieren (vgl. Valentin 2011: 80). Technikforschung nach Tixier (2012 [1978]: 39) heißt demnach eben nicht übergeordnete Zweckgebundenheiten zu erhellen oder metaphysische Sinnzusammenhänge freizulegen`, sondern die Bedingungen der Werkzeugherstellung zu ergründen. Der moderne chaîne opératoire-Ansatz, der in diesem intellektuellen Kontext für die Lithikforschung erst formuliert worden ist, versucht entsprechend technische Handlungen zu kartieren, um die den Fertigungsprozessen implizite `praktische Intelligenz` zu ergründen (vgl. Pelegrin/Karlin/Bodu1988).

Insbesondere Tixiers Schüler haben in der Folge den Versuch unternommen diese intime Verbindung von Technizität und Kognition näher zu untersuchen. Catherine Perlès (1974: 817) z.B. betont schon früh in einem Überblicksbeitrag zur Evolution menschlicher Steinartefakt-Technologie, dass sich die Hominisierung vor allem über die >reziproke Kopplung`von Werkzeuggebrauch und Kognitionsvermögen nachvollziehen lässt. Schon Perlès (1974: 816) konstatiert dabei - ganz im Sinne Bergsons - eine zunächst graduelle Differenz zwischen Mensch und Tier und definiert den Werkzeuggebrauch als notwendiges, keinesfalls aber hinreichendes Kriterium für die Menschwerdung. Ist der Mensch erst einmal Homo faber, erscheint eine Auseinanderentwicklung von Mensch und Tier aber unausweichlich - die graduellen Unterschiede münden in einer qualitativen Wesensverschiedenheit. Der Grund ist auch hier die sich in der evolutionären >Dauer radikalisierende Ko-Konstituierung von Mensch und Materie, die völlig neuartige Entwicklungspfade eröffnet und beide Protagonisten unabdingbar verändert.

Ausgehend von dieser wechselseitigen `Eingelassenheit` von Mensch und Technik, können Steinartefakt-Technologien in ihrer Virtualität beschrieben werden (vgl. Pelegrin 1995: 29-31). Interpretative Konzepte wie schéma opératoire, projet cognitif oder intention technique verweisen auf genau diese simmaterielle` Dimension lithischer Technologie. Die genannten Begriffe beschreiben >psycho-technischeく Eigenschaften oder Entitäten, welche zwar nicht physisch, aber doch in ihrer Funktionalität oder Wirkung vorhanden sind. Sie betreffen vor allem die `organisierenden Relationen`, die sich zwischen Mensch und Technik entfalten, wenn beide in ein interaktives Verhältnis treten und stabile Konfigurationen eingehen. Steinartefakt-Technologien können auf diese Weise als Ergebnis potenter Mensch-Materie-Wechselwirkungen verstanden werden. Sie zeugen von einer skreativen Bearbeitung von lithischen Rohmaterialien, die sich anhand von drei Konstitutivgrößen vollzieht: (1) Das ontogenetisch erworbene `Wissen, wie (savoir-faire), welches technische, soziale und individuelle Variabilität in der Steinbearbeitung abbildet 
(vgl. Ploux 1988); (2) das soziogenetisch erworbene >prä-reflexiv konzeptuelle Wissen (connaissance), welches die historischen Voraussetzungen der Mensch-Technik Schnittstelle behandelt (vgl. Pigeot 1987; Karlin 1991); und (3) die `technische Welt ` (milieu technique), welche die Gesamtheit der relevanten materiellen und psycho-technischen Apriori der Technikevolution festlegt und so die konkrete historische Ausprägung von Mensch-Technik-Relationen vermittelt (vgl. Pelegrin 2004).

Der resultierende Technologiebegriff ist dynamisch und betont die `Synchronisation von Mensch und Materie. Erst ein Verständnis dieser wechselseitigen Bezugnahme - der Mensch versucht die Materie zu seinen Gunsten zu gestalten, während die geformte Materie den Menschen zunehmend kognitiv vereinnahmt - erlaubt demnach einen gewinnbringenden Blick auf die übergeordneten Trends der Technikentwicklung in der Altsteinzeit. Dieser Auffassung liegt, wie wir gesehen haben, eine Homo faber Konzeption zugrunde, der zufolge der Mensch sein Humanpotenzial vorrangig herstellend verwirklichen kann. Jedwede technische Bezugnahme auf die Welt ist insofern immer schon `intelligenzschwanger`. Der ’kognitive` Ansatz der französischen chaîne-opératoire Forschung versucht, sich diese Tatsache zu Nutze zu machen (vgl. Karlin/Julien 1994; Pelegrin 2005; Pelegrin/Roche 2017). Die kognitive Verfasstheit von Hominiden und Frühmenschen wird über deren `Produktionsintelligenz`, und nicht etwa über deren Gehirngröße oder andere bio-physiologische Faktoren, erschlossen.

Diese herausragende Stellung der praktischen Intelligenz des Menschen kann durch zwei Details des französischen Diskurses weiter verdeutlicht werden. Erstens ist in der französischen Technologieforschung schon früh die Rolle der Ergonomie, der Wissenschaft von den Gesetzmäßigkeiten menschlicher Arbeit, herausgestellt worden (vgl. z.B. Perlès 1974: 818, 1991). Ein zentraler Aspekt dieser Forschungsperspektive ist die Identifizierung von Modalitäten der Handhabung lithischer Rohmaterialvolumen (Valentin 1995: Annexes [1-19]), die Auskunft über die >psycho-motorischen Implikationen bestimmter Mensch-Technologie Schnittstellen geben sollen. Zweitens sollte der `Technologe auch praktizierender Steinschläger sein, da nur so ein privilegierter Einblick in die Prinzipien der Bruchmechanik und die Konsequenzen spezifischer technischer Operationen gewonnen werden kann (Perlès 2016: 225). Diese Sichtweise rekapituliert die Logik des faber und betont den Erfahrungshorizont des `Produzenten`, welcher der theoretisch-reflexiven Kognition des Homo sapiens weitgehend entzogen bleibt.

\section{Faber-Peripetie: Boëda und Stiegler}

Ein vorläufiger Höhepunkt der >Faberisierung ` der frühen Menschheitsgeschichte ist in jüngerer Vergangenheit durch die Formulierung des `techno-genetischen Ansatzes` im Umfeld von Éric Boëda in Paris-Nanterre erreicht worden. Die Wurzeln dieser Perspektive, welche vor allem auf die Genesebedingungen technikgeschichtlicher Abstammungslinien (lignées) scharfzustellen versucht, liegen bereits in den 1990er Jahren. Während die methodischen Grundlagen dieses Ansatzes also schon relativ früh entwickelt worden sind (Boëda 1991a, 1991b; Bourguignon 1997), hat der theoretisch-konzeptuelle Rahmen erst vor Kurzem an Kontur und Kohärenz gewonnen (Boëda 1997, 2005, 2013). Technogenetische Forschung versucht durch eine Verschiebung des Untersuchungsschwerpunkts 
auf die longue durée der Technikevolution bisher kaum beachtete `Entwicklungstendenzen und deren übergeordnete >Techno-Logik ^freizulegen.

Der Protagonist dieses Ablegers der ursprünglichen `Technologie-Schule`, Éric Boëda, hat sich dabei dem intellektuellen Erbe des `frühen`André Leroi-Gourhan und des französischen Technikphilosophen Gilbert Simondon verschrieben. Beide Autoren knüpfen an die Lebensphilosophie Henri Bergsons an und haben darauf aufbauend jeweils ihre eigenen technikphilosophischen Perspektiven entwickelt. Beide importieren Bergsons Konzept des Homo faber und nehmen seine anti-Darwinistische Evolutionstheorie auf - eine Theorie, die, wie wir gesehen haben, das >organisch-poietischeく Potenzial evolutionärer Prozesse in den Mittelpunkt stellt. Boëda (2013) selbst zitiert Bergsons berühmt gewordenen Satz »wenn wir uns alles Stolzes entkleiden könnten, wenn wir uns, um unsere Art zu definieren, strikt an das halten würden, was Geschichte und Vorgeschichte uns als das konstante Charakteristikum des Menschen und der Intelligenz aufweisen, dann würden wir vielleicht nicht Homo sapiens, sondern Homo faber sagen « (Bergson 2013: 162) gleich zu Beginn von Techno-logique \& Technologie - ohne jeden Zweifel auch, um die Vorrangigkeit des faber für seinen Ansatz zu unterstreichen.

Das Zentrum der Bergsonschen Evolutionstheorie bilden Entwicklungszusammenhänge, die nur in der >Dauer ` (durée) erkennbar werden, gleichzeitig aber >gerichtet $>$ und >irreversibel sind. Im Frühwerk Leroi-Gourhans, Évolution et Techniques (1943/1945), wird dieses Moment mit dem Begriff der tendance eingefangen, während Simondon (vgl. 2012 [1958]) Konzepte wie `Individuierung` und `Konkretisierung « mobilisiert, um ähnliche Prozesse zu charakterisieren. Für Boëda bietet der resultierende Interpretationsrahmen jedenfalls die einmalige Chance, die Technikgeschichte des Menschen, so wie sie sich im paläolitharchäologischen Befund und damit in der Tiefenzeit darstellt, saus sich selbst heraus` (sui generis) verständlich zu machen. Das ist die autopoietische Implikation des techno-genetischen Denkens - eine Konsequenz, die typischerweise bei Fachkollegen, die sich dem Studium der Humanevolution im Sinne Darwins verschrieben haben, auf erhebliche Irritation stößt.

Der techno-genetische Ansatz ist damit aber auch mit einer zentralen Schwierigkeit konfrontiert. Es stellt sich nämlich die Frage, wie ein technikgeschichtlicher Entwicklungszusammenhang, eine >Abstammungslinie technischer Objekte, überhaupt bestimmt und definiert werden kann. Die Antwort auf diese Frage kann nur im Bereich des Funktionszusammenhangs eines technischen Systems selbst gefunden werden (efficacité). Sie muss, mit anderen Worten, mit der Funktionalität, oder genauer gesagt der `Techno-Funktionalität von Technik beantwortet werden (vgl. Boëda 2013: 33). Die Herausforderung technogenetischer Forschung liegt deshalb prinzipiell darin, den Organisationszusammenhang von Technik wieder mit dessen Funktionszusammenhang in Beziehung zu setzten und die schrittweise, aber kontinuierlich verlaufende Transformation von Organisationszusammenhängen mit weitgehend konstant bleibendem Funktionskreis in der longue durée zu dokumentieren. Insofern impliziert die techno-genetische Perspektive eine gewisse Überwindung von Tixiers Gründerprämissen und beschwört eine Re-Integration von ,Wie-` und `Was-Fragen ` in der Technikforschung herauf (vgl. Soriano 2000: 7). Der relativ stabile Funktionszusammenhang, der eine technikgeschichtliche Abstammungslinie 
ermöglicht, entspricht jedenfalls dem, was Simondon (2012 [1958]: 40) als »technische Essenz« bezeichnet hat.

Das Grundproblem von Boëdas Homo faber - und darin liegen zu einem großen Teil auch dessen `Gestaltungstrieb $<$ und `Eigenkreativität begründet - ist die generative Leistungssteigerung seiner Artefakte in sich stetig verändernden technischen und ökologischen Kontexten. Die Technizität des faber entwickelt sich somit nach dem Prinzip der Möglichkeit einer evolutionären Ausschöpfung 'funktionaler Artefakt-Potenzialität ‘ Diese Potenzialität ist eine verteilte, sie betrifft die poiesis des faber selbst, aber entfaltet sich auch über die strukturelle Bedingtheit seines technischen Milieus: es ist von einem gewissen `technikeigenen Widerstand ‘ auszugehen, der die Veränderungsrichtung sowie die Logik funktionaler Effizienzsteigerung in technischen Systemen mitreguliert (vgl. Boëda 2013: 42). Das ist die `ko-evolutive` Natur des Homo faber, auf die Boëda (vgl. 1997, 2005: 47, 63) so großen Wert legt - ein faber, der sich in der direkten Abarbeitung mit Materie kontinuierlich >re-aktualisiert und, wie Boëda sagt, »neu erfindet« $(1997,2005:$ 47, 63).

Im Anschluss an Simondon geht Boëda davon aus, dass sich Technikevolution nach dem Prinzip funktionaler `Sättigung ` vollzieht. Technische Objekte durchlaufen in ihrer Evolution interne Reorganisationsprozesse, die ihre funktionalen Teile auf ihre funktionalen Hauptaufgaben hin immer wieder neu einstellen (vgl. Boëda 2013: 55-58) - ein Vorgang, der als technikeigener \Lernprozess` verstanden werden kann. Grundsätzlich mündet dieses Modell jedenfalls in der allgemeinen Einsicht, dass technische Systeme dazu tendieren, ihre jeweiligen funktionalen Untereinheiten auf evolutionären Zeitskalen so zu integrieren, dass reziproke und synergetische Funktionsmodalitäten ausgebildet werden können. Das Ergebnis ist eine evolutionäre `Neigung〈, solche Funktionsweisen zu stabilisieren, innerhalb derer die funktionalen Teile eines technischen Objekts von dessen Funktionsgesamtheit nicht mehr zu trennen sind. Mit Simondon (2012 [1958]: 20) kann von einem Vorgang der »Konkretisierung " gesprochen werden, in dem technische Objekte sprichwörtlich zu sich selbst finden und dadurch erst »individuiert « werden - Simondon spricht in diesem Zusammenhang auch von techno-genetischer "Selbst-Anpassung" (Simondon 2012 [1958]: 20).

Um ebensolche evolutionären Prozesse funktionaler `Sättigung ‘ besser nachvollziehen zu können, hat die techno-genetische Forschung ein methodisches Instrumentarium zur Untersuchung sogenannter »Techno-Funktionaler-Einheiten« oder »UTFs « (unités techno-fonctionnelles) entwickelt (vgl. insb. Lépot 1993; Boëda 1997: 34-36). Grundsätzlich versucht dieser Ansatz zu klären, welchen Beitrag unterschiedliche Teile eines technischen Objekts zu dessen Gesamtfunktionalität leisten. Die UTF-Analyse versucht dabei zum einen, die relevanten Funktionseinheiten freizulegen und zu charakterisieren, zum anderen aber auch, die wechselseitige Verschränkung dieser Einheiten zu erhellen (vgl. z.B. Soriano 2000: Annexe II). Ohne hier ins Detail gehen zu können, sollte zumindest darauf hingewiesen werden, dass auf diese Weise zwischen ‘aktiven tefaktbereichen unterschieden und die Architektur modifizierter Werkzeugkanten auf ihr jeweiliges Funktionspotenzial hin untersucht werden kann. Methodisch macht sich die UTF-Analyse dabei zunutze, dass nicht jede Kantenmorphologie, nicht jeder Schneidewinkel und nicht jeder Kantenquerschnitt gleichermaßen geeignet ist, um bestimmten »Werkzeugbedürfnissen« (besoins techniques) nachzukommen. 
Der Vergleich des $>$ Integrationsgrads \&unktionaler Einheiten innerhalb verschiedener raumzeitlich verortbarer technischer Objekte erlaubt die Rekonstruktion von regionalspezifischen `Evolutionslinien` (lignées techniques), z.B. von sog. >Faustkeilen $($ vgl. Soriano 2001). Diese technischen Entwicklungspfade sind in der Regel nicht voraussagbar, sondern müssen diagnostiziert werden. Eine technische Abstammungslinie verständlich zu machen heißt zudem, sie mit Hilfe ihrer eigenen Entwicklungspotenziale - d.h. den der Evolutionslinie inhärenten ko-evolutiven Interaktionsdynamiken - zu rekonstruieren. sStabilität ‘ und >Veränderung ‘ menschlicher Technik werden nicht etwa durch externe Faktoren erklärt, sondern allein aus der >Innensicht $<$ nachvollzogen (vgl. Boëda 1997: 121-144). Technikexterne Faktoren wie z.B. Klima, Mobilität und Demographie werden demgegenüber, wenn überhaupt, nur dann als erklärende Faktoren in Betracht gezogen, wenn diese Binnenperspektive keine überzeugenden Argumente mehr zu liefern vermag (vgl. Boëda 2005, 2013: 231-236; Chevrier 2012: 98f., 115-120). Das Primat des Homo faber gilt hier also auch in erklärungsstrategischer Hinsicht.

Der techno-genetische Ansatz mündet also in der Freilegung von diskreten, aber zeitlich ausgedehnten technischen Abstammungslinien. Die Diversität dieser Abstammungslinien verweist zweifelsfrei auf die `technische Produktivität des Homo faber. Mit Boëda kann außerdem gesagt werden, dass jede Abstammungslinie sowohl eine spezifische >Aktualisierungsform ` als auch eine spezifische >Akkumulationsbahn` des faber anzeigt. Boëda bezieht sich hier vor allem auf Bernard Stiegler, einem der einflussreichsten zeitgenössischen Technikphilosophen Frankreichs, der die Kerngedanken von LeroiGourhan und Simondon entscheidend weiterentwickelt hat. Mit Stiegler (2004) lässt sich präzisieren, dass jeder einzelne dieser technischen Entwicklungszusammenhänge eine einzigartige Mensch-Materie Konfiguration abbildet. Die in technischen Abstammungslinien 'wirkende` Technizität verweist deshalb immer auch auf ein >epiphylogenetisches Gedächtnis (vgl. Stiegler 2009 [1994]: 234-236). Dieses Gedächtnis ist nichts anderes als das Ergebnis ko-evolutionär herausgebildeter `Produktionsintelligenz` und lässt so ganz unterschiedliche, aber immer technologisch vermittelte `Human-Lebensformen` erkennbar werden (ebd.). Ko-evolution wird hier als evolutionäre Koordinationsleistung fassbar. Das folgenreiche Resultat dieser >Faberisierung ‘ der frühesten Menschheitsgeschichte ist die radikale Pluralisierung der Menschheit in der Tiefenzeit. ${ }^{6}$ Jede Abstammungslinie (lignée) verweist auf eine alternative Artikulation von Mensch und Materie und damit auf einen alternativen Modus des Menschseins. Es liegt auf der Hand, dass die Frage nach dem `Menschen`selbst auf dieser Basis ganz neu gestellt werden muss.

6 Boëda identifiziert die sabgebrochenen` Abstammungslinien des Menschen als Überbleibsel eines weitgehend »verlorenen Gedächtnisses« (mémoire oubliée) (Boëda 2013: 226). Die Rekonstruktion der allgemeinen Typik dieses Gedächtnisses hat deshalb fundamentalanthropologische Relevanz und dient der Dokumentation »technischer Alterität« in der longue durée. 


\section{Resümee}

Die Gegenüberstellung von anglophon geprägtem `Modernitäts-Diskurs` und französisch geprägtem `Technologie-Diskurs h hat gezeigt, dass die Bedeutung des Homo faber sich in beiden paläolitharchäologischen Forschungskontexten stark unterscheidet. Die Frage nach der behavioural modernity des Menschen hat sich ohne jeden Zweifel dem Paradigma des Homo sapiens verschrieben und versucht die Einheit des modernen Menschen sowie den evolutionären Grenzübertritt zwischen Tier und Mensch archäologisch plausibel zu machen. Der faber-Begriff dient dort vor allem dazu, das Übergangsstadium zwischen Natur und Kultur zu charakterisieren. Die französische Lithikforschung hat sich demgegenüber dem Primat des Homo faber verpflichtet. Eine substantielle Konzeption des faber erlaubt dort die Thematisierung der Ko-Konstruktion von >Natur $<$ und $>$ Kultur $<$ über das Medium `Technik«. Diese Perspektive führt zur Pluralisierung der Menschheit in der Tiefenzeit und zu der zentralen Einsicht, dass die kognitive Evolution des Menschen vor allem eine Nachwehe der Technikevolution ist.

Insbesondere der positiv bestimmte und erweiterte Homo-faber Begriff erweist sich somit als möglicher Ausweg aus den Dualismen der Vergangenheit und autorisiert einen längst überfälligen, neuen Blick auf die `technische Produktivität ‘ des paläolithischen Menschen. Es scheint daher unbedingt geboten, die Konsequenzen eines >kreativ-poietischen Anthropos weiter am Quellenmaterial der Paläolitharchäologie auszuleuchten. Die theoretisch-konzeptuelle Arbeit am Konzept des faber kann dabei, so hofft der Verfasser gezeigt zu haben, einen wichtigen Beitrag leisten. ${ }^{7}$

Der Beitrag hat ein peer-review-Verfahren mit double blind-Standard durchlaufen.

7 Der Verfasser dankt den Herausgebern dieses Bandes für die willkommene Möglichkeit seine Überlegungen zum Homo faber darzulegen. Dieser Essay ist nicht zuletzt auch Produkt des langjährigen und stets anregenden Dialogs des Verfassers mit dem a.r.t.e.s. Research Lab der Universität zu Köln. Alle Diskussionspartner, Ideengeber und Kritiker seien an dieser Stelle dankend bedacht. 


\section{Literatur}

Audouze, Françoise (2002): »Leroi-Gourhan, a Philosopher of Technique and Evolution«. In: Journal of Archaeological Research 10: 4, 277-306.

BALfet, Hélène (1975): »Technologie«. In: Éléments d'ethnologie, vol. 2, hg. v. Robert Cresswell, Paris: Armand Colin, 44-79.

BALfET, Hélène (Hg.) (1991): Observer l'action technique. Des chaînes opératoires pour quoi faire?, Paris: Éditions du C.N.R.S.

Beaune, Sophie de (2000): Pour une archéologie du geste, Paris: C.N.R.S. Éditions.

BEAUne, Sophie de (2011): "La genèse de la technologie comparée chez André Leroi-Gourhan. Introduction à son article `L'homme et la nature`, paru dans L'Encyclopédie française en 1936«. In: Documents pour l'histoire des techniques 20, 197-202.

Bergson, Henri (1944 [1907]): Creative Evolution, übers. v. Arthur Mitchell, New York: Random House.

Bergson, Henri (2013): Schöpferische Evolution, übers. v. Margarethe Drewsen, Hamburg: Felix Meiner.

BoËDA, Éric (1991a): »La conception trifaciale d'un nouveau mode de taille préhistorique«. In: Les premiers Européens, hg. v. Eugène Bonifay/Bernard Vandermeersch, Paris: Comité des travaux historiques et scientifiques, 251-263.

BoËDA, Éric (1991b): »Approche de la variabilité des systèmes de production lithique des industries du paléolithique inférieur et moyen: Chronique d'une variabilité attendue «. In: Technique \& Culture 17-18, 37-79.

Bö̈DA, Éric (1997): Technogenèse du système de production lithique au Paléolithique inférieur et moyen en Europe occidentale et au Proche-Orient, Unveröffentlichte Habilitationsschrift, Universität Paris X-Nanterre.

BoËDA, Éric (2005): „Paléo-technologie ou anthropologie des techniques?«. In: Arob@se 1, 46-64.

BoËDA, Éric (2013): Techno-logique \& technologie. Une Paléohistoire des objets lithiques tranchants, Paris: @rchéo-éditions.

Bourguignon, Laurence (1997): Le Moustérien de type Quina: nouvelle définition d'une entité technique, Unveröffentlichte Doktorarbeit, Universität Paris X-Nanterre.

BrIL, Blandine (1984): "Description du geste technique: Quelles méthodes?«. In: Techniques \& Culture 3, 81-96.

BRIL, Blandine/Roux, Valentine (2002): "Regards croisés sur le geste technique«. In: Le geste technique. Réflexions méthodologiques et anthropologiques, hg. v. Blandine Bril/Valentine Roux, Ramonville Saint-Agne: Éditions érès, 7-11.

CAssirer, Ernst (2007): Versuch über den Menschen. Einführung in eine Philosophie der Kultur, übers. v. Reinhard Kaiser, Hamburg: Felix Meiner.

Chevrier, Benoît (2012): Les assemblages à pièces bifaciales au Pléistocène inférieur et moyen ancien en Afrique de l'Est et au Proche-Orient Nouvelle approche du phénomène bifacial appliquée aux problématiques de migrations, de diffusion et d'évolution locale, Unveröffentlichte Doktorarbeit, Universität Paris X-Nanterre.

Conard, Nicholas (2010): „Cultural Modernity: Consensus or Conundrum?«. In: Proceedings of the National Academy of Sciences of the United States of America 107: 17, 7621-7622. 
Delitz, Heike (2015): Bergson-Effekte. Aversionen und Attraktionen im französischen soziologischen Denken, Weilerswist: Velbrück Wissenschaft.

Eliade, Mircea (1959): »Methodological Remarks on the Study of Religious Symbolism«.

In: The History of Religions: Essays on Methodology, hg. v. Joseph Kitagawa/Mircea Eliade, Chicago: University of Chicago Press, 86-107.

Grawe, Christian (1973a): »Homo sapiens«. In: Historisches Wörterbuch der Philosophie $B d$. 3, hg. v. Joachim Ritter/Karlfried Gründer/Gottfried Gabriel, Basel: Schwabe Verlag, 1178-1179.

Grawe, Christian (1973b): »Homo faber«. In: Historisches Wörterbuch der Philosophie Bd. 3, hg. v. Joachim Ritter/Karlfried Gründer/Gottfried Gabriel, Basel: Schwabe Verlag, 1173-1175.

Haudricourt, André-Georges (1987): La technologie, science humaine. Recherches d'histoire et d'ethnologie des techniques, Paris: Édition de la Maison des Sciences de l'Homme.

Heilmann, Till (2016): » Zur Vorgängigkeit der Operationskette in der Medienwissenschaft und bei Leroi-Gourhan«. In: Internationales Jahrbuch für Medienphilosophie 2: 1, 7-30.

Henshildwood, Christopher/D'Errico, Francesco (Hg.) (2011): Homo Symbolicus. The Dawn of Language, Imagination and Spirituality, Amsterdam: John Benjamins Publishing. InIZAN, Marie-Louise/Reduron-Ballinger, Michèle/Roche, Hélène/Tixier, Jacques (1995): Technologie de la pierre taillée, Paris: C.R.E.P.

Johnson, Christopher (2011): »Leroi-Gourhan and the Limits of the Human«. In: French Studies LXV: 4, 471-487.

KARLIN, Claudine (1991): „Connaissances et savoir-faire: comment analyser un processus technique en préhistoire, introduction«. In: Treballs d’Arqueologia 1, 99-124.

Karlin, Claudine/Julien, Michèle (1994): Prehistoric Technology: A Cognitive Science?«. In: The Ancient Mind: Elements of a Cognitive Archaeology, hg. v. Colin Renfrew/Ezra Zubrow, Cambridge: Cambridge University Press, 152-164.

Kelly, Robert (2016): The Fifth Beginning. What Six Million Years of Human History Can Tell Us About Our Future, Oakland: University of California Press.

KNight, Chris (2010): »The Origins of Symbolic Culture«. In: Homo Novus - A Human Without Illusions, hg. v. Ulrich Frey/Charlotte Störmer/Kai Willführ, Berlin: Springer, 193-211.

Knight, Chris/Power, Camilla/Watts, Ian (1995): »The Human Symbolic Revolution: A Darwinian Account«. In: Cambridge Archaeological Journal 5: 1, 75-114.

Lemonnier, Pierre (2012): Mundane Objects. Materiality and Non-verbal Communication, Walnut Creek: Left Coast Press.

LEPOT, Michel (1993): Approche techno-fonctionnelle de l'outillage lithique moustérien: essai de classification des parties actives en termes defficacité technique. Application à la couche M2e sagittale du Grand Abri de La Ferrassie (fouille Henri Delporte), Unveröffentlichte Mémoire de la Maitrise, Universität Paris X-Nanterre.

Leroi-Gourhan, André (1943): Évolutions et techniques: L'homme et la matière, Paris: Éditions Albin Michel.

Leroi-Gourhan, André (1945): Évolutions et techniques: Milieu et technique, Paris: Éditions Albin Michel. 
Leroi-Gourhan, André (1949): »Note sur les rapports de la technologie et de la sociologie«. In: L’Année sociologique 3ième série: 2, 766-772.

Leroi-Gourhan, André (1952): »Homo faber - Homo sapiens«. In: Revue de Synthèse 30, 79-102.

Leroi-Gourhan, André (1953): „L'homo faber: la main«. In: A la recherche de la mentalité préhistorique, hg. v. Annette Laming, Paris: Éditions Albin Michel, 75-98.

Leroi-Gourhan, André (1984 [1964/1965]): Hand und Wort. Die Evolution von Technik, Sprache und Kunst, übers. v. Michael Bischoff, Frankfurt/Main: Suhrkamp.

Mauss, Marcel (2012 [1935]): »Les techniques du corps«. In: Marcel Mauss: Techniques, technologie et civilisation, hg. v. Nathan Schlanger, Paris: Presses Universitaires de France, 365-394.

Mellars, Paul (1991): "Cognitive Changes and the Emergence of Modern Humans in Europe«. In: Cambridge Archaeological Journal 1, 763-776.

Mellars, Paul /Stringer, Chris (Hg.) (1989): The Human Revolution: Behavioral and Biological Perspectives on the Origins of Modern Humans, Edinburgh: Edinburgh University Press.

Mellars, Paul/Boyle, Katherine/Bar-Yosef, Ofer (Hg.) (2007): Rethinking the Human Revolution: New Behavioural and Biological Perspectives on the Origin and Dispersal of Modern Humans, Cambridge: McDonald Institute for Archaeological Research.

Mithen, Steven (1998): The Prehistory of the Mind. A Search for the Origins of Art, Religion and Science, London: Orion Books.

Nowell, April (2010): »Defining Behavioral Modernity in the Context of Neandertal and Anatomically Modern Human Populations«. In: Annual Review of Anthropology 39, 437-452.

Pelegrin, Jacques (1991): »Les savoir-faire: une très longue histoire«. In: Terrain, Carnets du Patrimoine Ethnologique 6, 106-113.

Pelegrin, Jacques (1995): Technologie lithique: le Châtelperronien de Roc-de-Combe (Lot) et de La Côte (Dordogne), Paris: Éditions de C.N.R.S.

Pelegrin, Jacques (2004): »Le milieu intérieur d'André Leroi-Gourhan et l'analyse de la taille de pierre au Paléolithique«. In: Autour de l'homme. Contexte et actualité d'André Leroi-Gourhan, hg. v. Françoise Audouze/Nathan Schlanger, Antibes: Éditions A.P.D.C.A., 149-160.

Pelegrin, Jacques (2005): »Remarks About Archaeological Techniques and Methods of Knapping: Elements of a Cognitive Approach to Stone Knapping «. In: Stone Knapping, the Necessary Conditions for a Uniquely Hominin Behavior, hg. v. Valentine Roux/Blandine Bril, Cambridge: McDonald Institute for Archaeological Research, 23-34.

Pelegrin, Jacques/Karlin, Claudine/Bodu, Pierre (1988): „Chaînes opératoires: un outil pour le prehistorien«. In: Technologie préhistorique, hg. v. Jacques Tixier, Paris: Éditions du C.N.R.S., 55-62.

Pelegrin, Jacques/Roche, Hélène (2017): »L'humanisation au prisme des pierres taillées«. In: Comptes Rendus Palevol 16: 2, 175-181.

Perlès, Catherine (1974): »Importance et évolution de l'outil préhistorique«. In: Archives des Maladies Professionnelles 35: 9, 816-818. 
PerLÈs, Catherine (1991): "Introduction«. In: 25 Ans détude technologique en préhistoire. Bilan et perspectives, hg. v. Catherine Perlès, Juan-les-Pins: Éditions APDCA, 7-10.

Perlès, Catherine (2016): »La technologie lithique, de part et d'autre de l'Atlantique/Paths that Rarely Cross: Approaches to Lithic Technology Across the Atlantic«. In: Bulletin de la Société préhistorique française 113: 2, 221-240.

Pigeot, Nicole (1987): Magdaléniens d'Etiolles. Économie de débitage et organisation sociale, Paris: Éditions du C.N.R.S.

Pigeot, Nicole (1991): »Réflexions sur l'histoire technique de l'Homme: de lévolution cognitive à lévolution culturelle«. In: Paléo 3, 167-200.

Ploux, Sylvie (1988): Approche archéologique de la variabilité des comportements individuels. Lexemple de quelques tailleurs magdaléniens à Pincevent, Unveröffentlichte Doktorarbeit, Universität Paris X-Nanterre.

Renfrew, Colin (2009): Prehistory. The Making of the Human Mind, New York: Modern Library.

Renfrew, Colin/Morley, Ian (Hg.) (2009): Becoming Human. Innovation in Prehistoric Material and Spiritual Culture, Cambridge: Cambridge University Press.

SCHLANGer, Nathan (2004): "Suivre les gestes, éclat par éclat - la chaîne opératoire d'André Leroi-Gourhan«. In: Autour de l'homme. Contexte et actualité d'André LeroiGourhan, hg. v. Françoise Audouze/Nathan Schlanger, Antibes: Éditions A.P.D.C.A., 5-25.

Schlanger, Nathan (2015): "L'insaisissable technologie d'André Leroi-Gourhan. Des tendances et des faits des années 1930 à l'après-guerre«. In: André Leroi-Gourhan, »l'homme simplement«, hg. v. Philippe Soulier, Paris: Éditions de Boccard, 103-116.

SснӥтtPELZ, Erhard (2006): „Die medienanthropologische Kehre der Kulturtechniken«. In: Archiv für Mediengeschichte 6, 87-110.

Sigaut, François (2012): Comment Homo devint faber. Comment l'outil fit l'homme, Paris: C.N.R.S. Éditions.

Simondon, Gilbert (2012 [1958]): Die Existenzweise technischer Objekte, übers. v. Michael Cuntz, Zürich: diaphanes.

Soriano, Sylvain (2000): Outillage bifacial et outillage sur éclat au Paléolithique ancien et moyen: coexistence et interaction, Unveröffentlichte Doktorarbeit, Universität Paris XNanterre.

Soriano, Sylvain (2001): "Statut fonctionnel de l'outillage bifacial dans les industries du Paléolithique moyen. Propositions méthodologiques«. In: Industries à outils bifaciaux du Paléolithique moyen d'Europe occidentale, hg v. Dominique Cliquet, Liège: ERAUL 98, 77-83.

STIEgler, Bernard (2004): »André Leroi-Gourhan et la raison technologique. Lépiphylogenèse«. In: Autour de l'homme. Contexte et actualité d'André Leroi-Gourhan, hg. v. Françoise Audouze/Nathan Schlanger, Antibes: Éditions A.P.D.C.A., 69-92.

StIEgler, Bernard (2009 [1994]): Technik und Zeit. Der Fehler des Epimetheus, übers. v. Gabriele Ricke und Ronald Voullié, Zürich: diaphanes.

Stringer, Chris (2006): Homo Britannicus: The Incredible Story of Human Life in Britain, London: Penguin Books. 
Tixier, Jacques (1980): »Avant-Propos«. In: Préhistoire et technologie lithique, 11-13 mai 1979, vol 1, Paris: Centre régional de publication de Sophia Antipolis 5.

Tixier, Jacques (2012 [1978]): A Method for the Study of Stone Tools/Méthode pour létude des outillages lithiques, hg. v. Foni Le Brun-Ricalens und übers. v. Marie-Claire Dawson und Brad Gravina, Luxemburg: ArchéoLogique 4.

Тотн, Nicholas/Schick, Kathy (2009): »The Oldowan: The Tool Making of Early Hominins and Chimpanzees Compared«. In: Annual Review of Anthropology 38, 289-305.

Toth, Nicholas/Schick, Kathy/Savage-Rumbaugh, Sue/Sevcik, Rose/Rumbaugh, Duane (1993): »Pan the Tool-Maker: Investigations into the Stone Tool-Making and ToolUsing Capabilities of a Bonobo (Pan paniscus) «. In: Journal of Archaeological Science 20, 81-91.

VALENTIN, Boris (2008): Jalons pour une paléohistoire des derniers chasseurs (XIVe-VIe millénaire avant J.-C.), Paris: Publications de la Sorbonne.

VAlentin, Boris (2011): Le Paléolithique, Paris: PUF.

WATTs, Ian (2010): »Was there a Human Revolution?«. In: Radical Anthropology 4, 16-21. 